OPEN ACCESS

Edited by:

Fangliang Zhang,

University of Miami, United States

Reviewed by:

Vladimir N. Uversky,

University of South Florida,

United States

Lianjun Zhang,

Chinese Academy of Medical

Sciences, China

*Correspondence:

Anna Kashina

akashina@upenn.edu

Specialty section:

This article was submitted to

Cellular Biochemistry,

a section of the journal

Frontiers in Cell and Developmental

Biology

Received: 01 November 2021

Accepted: 06 January 2022

Published: 11 February 2022

Citation:

Vedula P, Tang H-Y, Speicher DW and Kashina A (2022) Protein Posttranslational Signatures Identified in COVID-19 Patient Plasma.

Front. Cell Dev. Biol. 10:807149.

doi: 10.3389/fcell.2022.807149

\section{Protein Posttranslational Signatures Identified in COVID-19 Patient Plasma}

\author{
Pavan Vedula ${ }^{1}$, Hsin-Yao Tang ${ }^{2}$, David W. Speicher ${ }^{2}$ and Anna Kashina ${ }^{1 *}$ \\ The UPenn COVID Processing Unit \\ ${ }^{1}$ Department of Biomedical Sciences, University of Pennsylvania School of Veterinary Medicine, Philadelphia, PA, United States, \\ ${ }^{2}$ The Wistar Institute, Philadelphia, PA, United States
}

Severe acute respiratory syndrome coronavirus-2 (SARS-CoV-2) is a highly contagious virus of the coronavirus family that causes coronavirus disease-19 (COVID-19) in humans and a number of animal species. COVID-19 has rapidly propagated in the world in the past 2 years, causing a global pandemic. Here, we performed proteomic analysis of plasma samples from COVID-19 patients compared to healthy control donors in an exploratory study to gain insights into protein-level changes in the patients caused by SARS-CoV-2 infection and to identify potential proteomic and posttranslational signatures of this disease. Our results suggest a global change in protein processing and regulation that occurs in response to SARS-CoV-2, and the existence of a posttranslational COVID-19 signature that includes an elevation in threonine phosphorylation, a change in glycosylation, and a decrease in arginylation, an emerging posttranslational modification not previously implicated in infectious disease. This study provides a resource for COVID-19 researchers and, longer term, and will inform our understanding of this disease and its treatment.

Keywords: COVID-19, proteomics, peptidomics, posttranslational modifications, arginylation

\section{KEY POINTS}

1. Plasma from COVID-19 patients exhibits prominent protein- and peptide-level changes

2. Proteins from COVID-19 patient plasma exhibit prominent changes in several key posttranslational modifications

\section{INTRODUCTION}

Severe acute respiratory syndrome coronavirus-2 (SARS-CoV-2) is a respiratory virus of the coronavirus family that causes coronavirus disease-19 (COVID-19) in humans and a number of animal species (Swelum et al., 2020). COVID-19 has rapidly propagated worldwide in the past 2 years, causing a global pandemic (see, e.g., (Novelli et al., 2021), for a recent review). This highly contagious disease causes respiratory symptoms that range from mild to severe, and is associated with a number of other serious health implications, including lung inflammation and damage, thrombosis, stroke, renal failure, neurological disorders, and others (Hanff et al., 2020; Schmulson et al., 2020; Troyer et al., 2020; Vakil-Gilani and O'Rourke, 2020; Harapan and Yoo, 2021; Ostergaard, 2021). This list continues to grow, and despite extensive research in the past year and a half, full understanding of COVID-19 mechanisms of action and health consequences has not yet been achieved. 
While the initial route of SARS-CoV-2 infection involves the respiratory tract, some of the most prominent effects of COVID19 can be detected in the blood plasma, which contains antibodies against SARS-CoV-2, and is a major site of immune response that builds the body's defense against the virus. It is of no coincidence that convalescent plasma from COVID-19 patients has been proposed as treatment for this disease, as well as a foundation for some of the diagnostic tests. Plasma is arguably one of the most rapidly changing environments upon SARS-CoV-2 infection. Thus, studies of the disease-related changes in the plasma appear highly promising as a tool that would enable better understanding of this disease progression.

Here, we performed proteomic analysis of plasma samples from COVID-19 patients with symptoms severe enough to require hospitalization compared to healthy control donors, in an attempt to gain insights into protein-level changes in the patients caused by SARS-CoV-2 infection and to identify potential proteomic and posttranslational signatures of this disease. Our analysis revealed a number of changes in protein and peptide composition of the COVID-19 patients' plasma samples. Furthermore, global analysis of posttranslational modifications (PTMs) in these samples showed a striking change in several key physiological PTMs, including phosphorylation, glycosylation, citrullination, and arginylation, which exhibited differential up- and down-regulation in COVID19 patients compared to controls and, in the case of arginylation and phosphorylation, modified different repertoire of sites on a limited number of target proteins. These patterns suggest a global change in protein processing and regulation that occurs in response to SARS-CoV-2, and the existence of a posttranslational COVID-19 “code". Deciphering this code may advance our understanding of disease progression and long-term implications, as well as potentially inform novel strategies of COVID-19 diagnostics and treatment.

\section{RESULTS AND DISCUSSION}

\subsection{COVID-19 Patients Exhibit Prominent Changes in Their Plasma Peptidomes}

To address potential protein and peptide changes in the plasma associated with COVID-19, we obtained plasma samples from 6 COVID patients with severe disease that required hospitalization and 7 similarly drawn control samples from healthy donors collected independently within the same time frame (Supplementary Table S1).

To analyze plasma peptidomes, we used size exclusion under denaturing conditions followed by $\mathrm{C} 18$ reverse phase cleanup to isolate plasma peptides followed by LC-MS/MS without proteolysis treatment. LC-MS/MS data were searched against the human protein database using no-enzyme specificity so that peptides naturally occurring or produced by in vivo proteolysis could be identified. The final search results were filtered by $p$-value $(<0.05)$ and fold change ( 2 -fold and above) to define 180 peptides that showed significant differences in abundance between patients and controls. The significantly changed peptides are shown in Supplementary Table S2, and the list of identified peptides that did not meet these statistical criteria and were not used in the final analysis is shown in Supplementary Table S3.

No known regulatory peptides were identified, likely due to their lower abundance. All the significant peptides identified constituted proteolytic fragments predominantly from abundant plasma proteins, which were apparently produced by proteolytic events that accompany immune response, cell migration and adhesion, and other physiological processes (Arapidi et al., 2018). Interestingly, when the total number of identified peptides were compared (Supplementary Tables S2, S3), substantially fewer peptides were identified overall in the COVID-19 samples compared to control (Supplementary Figure S1), in seeming contrast to the fact that SARS-CoV-2 infection is associated with increased proteolysis (Anand et al., 2020; Ramos-Guzmán et al., 2020; Świderek and Moliner, 2020; Meyer et al., 2021; Tang et al., 2021).

To further analyze COVID-19-dependent peptidomics trends, we used our significantly changed peptide list (Supplementary Table S2) and grouped the identified peptides by their parent proteins. For each given protein we plotted combined intensities of all significantly changed peptides (Supplementary Figure S2), and separately plotted all the combined intensities grouped by protein for the most abundant hits showing overall increase in control (Figure 1A) and COVID-19 (Figure 1B).

Most of the combined peptide intensities for each protein were substantially higher in the control samples compared to COVID19 , consistent with the fact that control plasma contained more peptides overall (Supplementary Figure S1). However, peptides derived from a small group of proteins showed significant elevation in COVID-19 plasma (Figure 1B). The most abundant of these, Alpha-1-antitrypsin, C4a anaphylatoxin, and Serum amyloid A-2, have known functional association with disease pathology. Alpha1 -antitrypsin is a protease inhibitor, involved in regulation of plasma proteolysis (Stockley, 2014). C4 anaphylatoxin and Serum amyloid A-2 are involved in immune response (Gorski et al., 1979; Zheng et al., 2020). All of these processes are highly relevant to the COVID-19 disease (Katneni et al., 2020; GómezMesa et al., 2021; Montenegro et al., 2021; Ostergaard, 2021), and thus increased abundance of significantly changed peptides for these proteins in COVID-19 patients may indicate a direct association between these proteins' proteolysis and SARS-CoV-2 infection. In contrast, peptide groups showing higher levels in control belong to normal proteins expected to be proteolyzed in the blood due to normal organismal functions, and their decrease in COVID-19 would likely lead to impairment of these functions because of SARS-CoV-2 infection.

An additional interesting observation concerns alphafibrinogen, which had the largest number of significantly changing peptides (Supplementary Table S2). Even though the combined intensities of significantly changed fibrinogenderived peptides was much higher in control compared to COVID-19 (Figure 1A; Supplementary Figure S2), individual peptides derived from alpha-fibrinogen showed different trends, with some peptides elevated in COVID-19 rather than control samples (Figure 2A; Supplementary Figure S2). The peptides with the highest abundance were still more prevalent in control (Figure 2A, left), however out of the 17 peptides showing significant change, 8 were more 

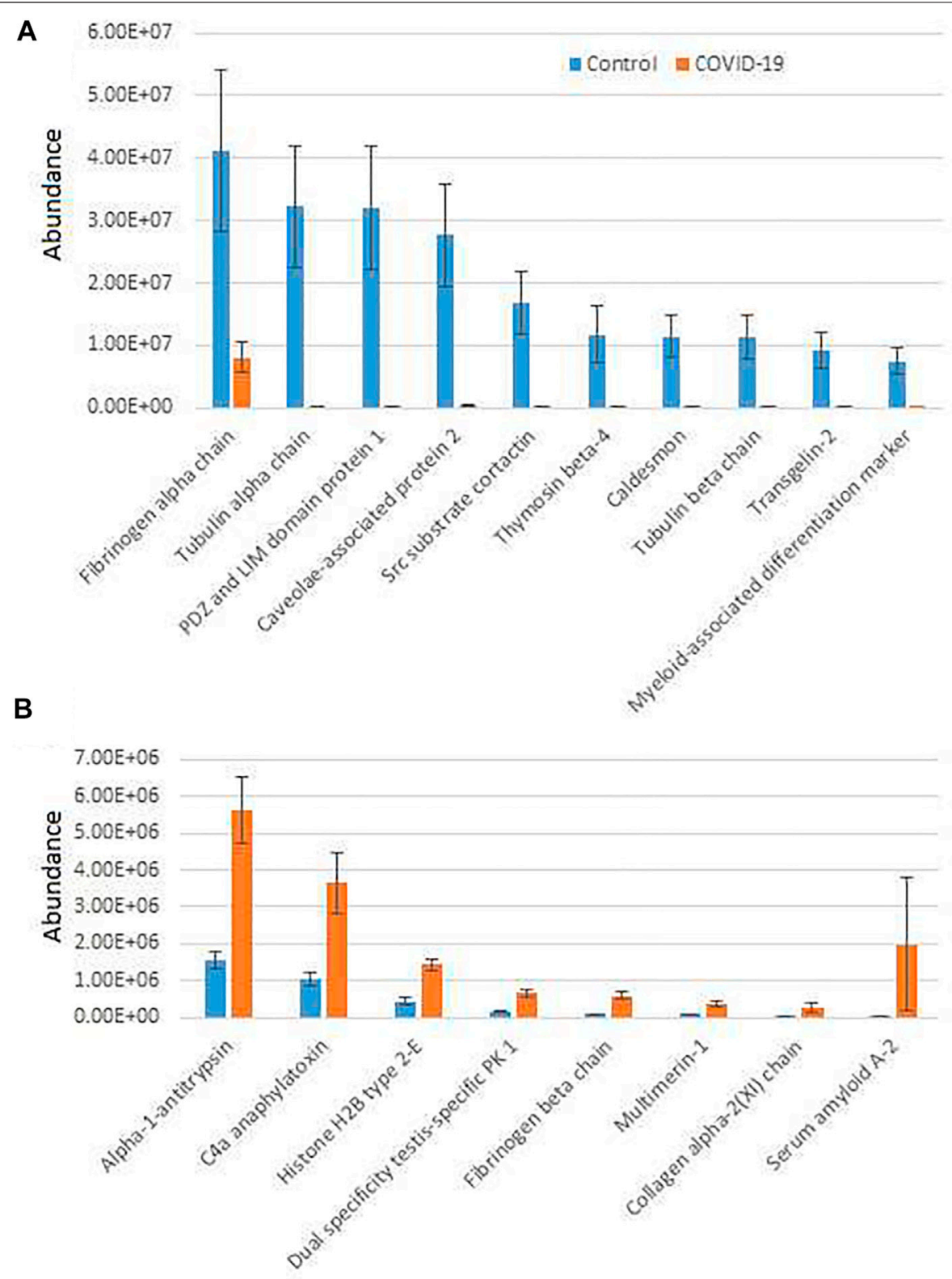

FIGURE 1 | Plasma peptides from COVID-19 patients exhibits prominent changes compared to control. Combined total intensities of all significantly changed peptides for each parent protein listed on the X axis. A and B show the most abundant peptide groups in control (A) and COVID-19 (B). See Supplementary Figure S2 for the full list of proteins with significantly changed peptides. Error bars represent SEM ( $n=7$ for control, 6 for COVID-19).

abundant in the COVID-19 samples (Figure 2A, right). A somewhat similar pattern was observed with serglycin: only two peptides were identified, but one of these was much more abundant in control, while the other showed the opposite trend (Figure 2B). While each change was significant, added together, the intensities of these peptides evened out and showed no change in the serglycin protein group shown in Supplementary Figure S2. This observation suggests that fibrinogen and serglycin undergo different proteolytic events in normal physiology and during SARS-CoV-2 infection. Fibrinogen plays a key role in blood clotting (May et al., 2021), a process shown to be impacted in
COVID-19 patients (Ahmed et al., 2020); serglycin is key to the biology of the blood cells (Scully et al., 2012). Altered proteolytic patterns of these proteins in COVID-19 may prove to be a potentially interesting biomarker in future studies.

\subsection{COVID-19 Patient Plasma Exhibits Prominent Changes in the Global Proteome} Next, we analyzed plasma samples by shotgun proteomics. For this, IgG/albumin-depleted plasma samples were loaded onto SDS PAGE and run $\sim 0.5 \mathrm{~cm}$ into the gel, followed by Coomassie Blue staining and excision of the entire protein-containing gel 
A

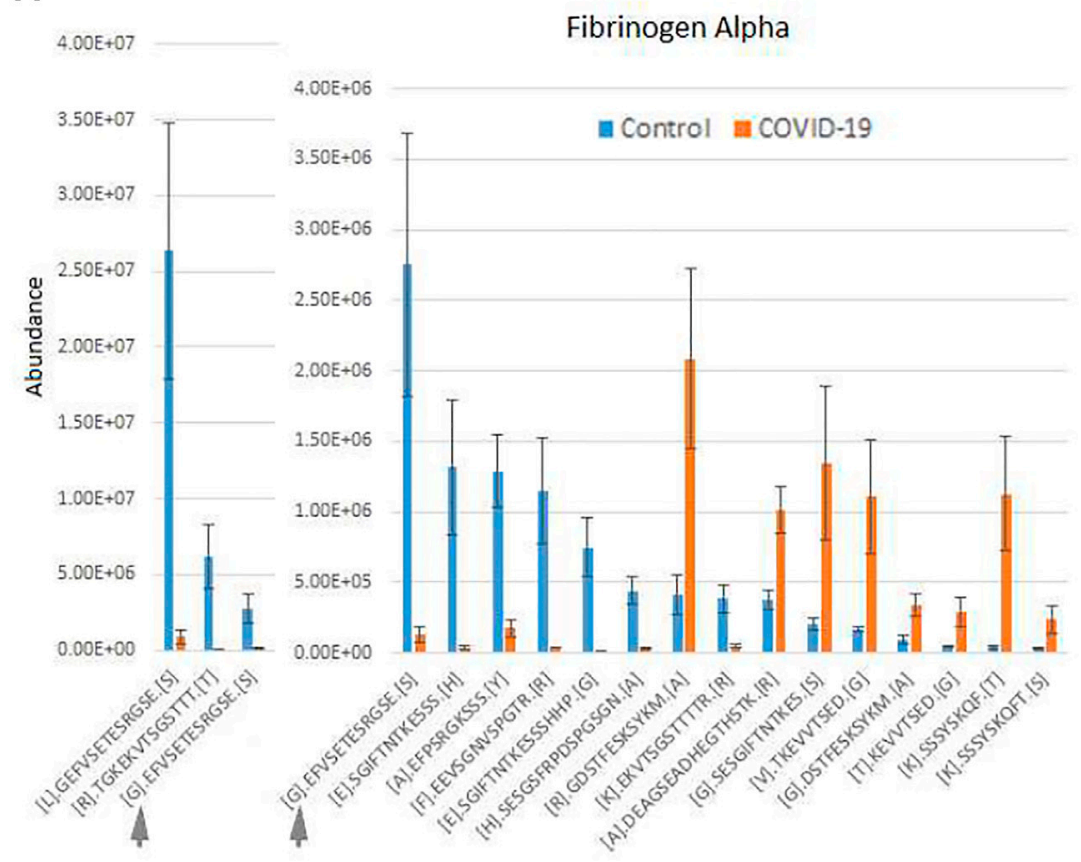

B

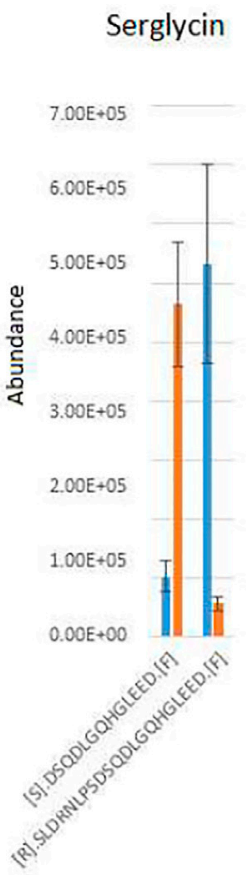

FIGURE 2 | Fibrinogen- and serglycin-derived peptides exhibit differential abundance changes between COVID-19 and control, suggesting different proteolytic patterns in response to Sars-CoV-2 infection. Normalized intensities of the most abundant individual peptides in control and COVID-19 plasma samples are shown for fibrinogen (A) and serglycin (B). Peptides in A are plotted in two charts on two different scales, including one overlapping peptide is shown in both charts for scale ([G]. EFVSETESRGSE[S] indicated with a gray arrow in both charts). Error bars represent SEM ( $n=7$ for control, 6 for COVID-19).

zone, which was then subjected to in-gel digestion with trypsin and analyzed by LC-MS/MS. Differences between COVID-19 and control samples were considered as high confidence significant changes if they exhibited a greater than 2-fold increase or decrease and q-value less than 0.05. Proteins that passed these criteria and were used for further analysis are listed in Supplementary Table S4. The remaining identified proteins are listed in Supplementary Table S5.

Interestingly, only 12 proteins were significantly increased in COVID-19 plasma while 35 proteins were decreased in these samples compared with controls (Figure 3; Supplementary Figure S3). When total iBAQ was used as a rough metric of relative abundance across proteins, only three of the 8 most abundant proteins were increased in COVID-19 plasma (Figure 3). One of these proteins, serum amyloid A-2 also showed up in our peptidomics dataset (Figure 1), where peptides derived from this protein were elevated to a similar extent in COVID-19 compared to control. This suggests that serum amyloid A-2 is both upregulated and more heavily proteolyzed in COVID-19. The other two proteins increased in COVID-19 plasma were serum amyloid A-1 and C-reactive protein, which are known to be elevated in the plasma in response to inflammation, and thus their increased levels observed in our dataset is fully consistent with known COVID-19 effects. In contrast, proteins showing decreased levels in COVID-19 compared to control (Figure 3; Supplementary Figure S3; Supplementary Table S4) are mostly related to normal physiological functions, including anti-inflammatory response (apolipoprotein A-IV and C-III) and overall protective functions (serum paraoxonase), hormone and vitamin transport (retinol-binding protein and transthyretin). It is possible that their elevated levels in control versus patient plasma reflect down-regulation or depletion of these normal proteins upon SARS-CoV-2 infection that ultimately contribute to disease pathology.

Thus, our data suggest that a limited set of proteins related to inflammation, immune response, and normal organismal homeostasis are prominently altered between COVID-19 and control, potentially as a direct consequence of SARS-CoV-2 infection.

\subsection{COVID-19 Plasma Proteome Exhibits Altered Posttranslational Modification Patterns}

To test whether COVID-19 is associated with any changes in PTMs, we analyzed our total proteomics run of plasma samples using pFIND, a software package that can simultaneously identify a large number of different PTMs, and chemical modifications based on mass shifts (Li et al., 2005; Wang et al., 2007). We manually added arginylation into the program (including addition of unmodified, mono- and dimethyl-Arg that have been all shown to occur in vivo through our previous work (Saha et al., 2011; Wang et al., 2014; Wang et al., 2019)). This 


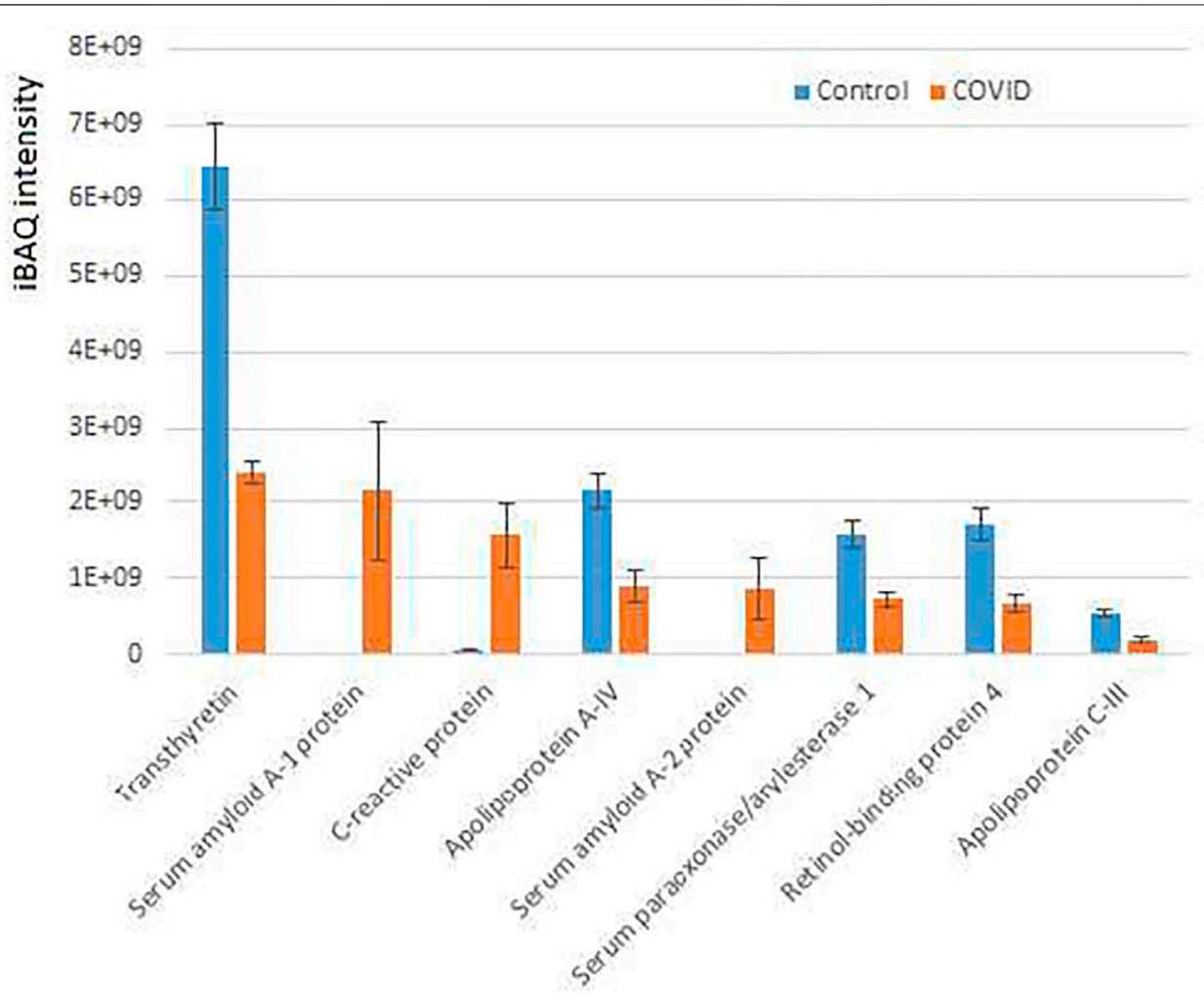

FIGURE 3 | Plasma proteins from COVID-19 patients exhibits prominent changes compared to control. iBAQ intensities of the most abundant proteins showing significant differences between COVID-19 and control. See Supplementary Figure S4 for the full list of hits. Bars represent normalized intensity levels averaged for all samples in each group, error bars represent SEM ( $n=7$ for control, 6 for COVID-19).

search identified a total of 2630 modifications in the samples. The results were filtered by precursor mass tolerance of $10 \mathrm{ppm}$, fragment ion tolerance of $20 \mathrm{ppm}$, and false discovery rate $(\mathrm{FDR})<1 \%$ at peptide and protein level.

Total results from the pFIND search, including data in individual samples, is shown in Supplementary Table S6, and the 10 most abundant modifications from COVID-19 and control patient samples are plotted in Supplementary Figure S4. These results were used to calculate each modified peptide amount as spectra count normalized to the percent of the total peptides in the sample, and these numbers were then compared across samples between control and COVID-19 to calculate $p$-value and fold change.

We defined putative significant differences as a greater than 1.5 fold change between control and COVID-19 peptides, with $p$-value less than 0.05. These hits are listed in Supplementary Table S6, and the modified sites identified in our search are listed in Supplementary Table S7.

A total of 82 PTMs showed statistically significant differences between COVID-19 and control. However, many of these modifications are apparently chemically induced and have not been described to happen under normal physiological conditions. Such modifications in the plasma can potentially occur in response to drugs and environmental factors; thus, it is unlikely that these modifications occur because of SARS-CoV2 infection, even though they might be directly or indirectly related to the patients' treatment or susceptibility to symptomatic COVID-19. Given this uncertainty, we excluded these PTMs, and manually selected only the known naturally occurring modifications for further analysis.

After this filtering, only a few physiological PTMs showed significant differences between COVID-19 and controls (Figure 4). These PTMs were plotted in three separate groups: high abundance (Figure 4A), intermediate abundance (Figure 4B), and low abundance (Figure 4C). Notably, only three of them were in the high abundance group, including phosphorylation on Thr, which showed a nearly 2-fold increase in COVID-19 patients, Arg deamidation, $\sim 2$-fold increased in control, and a $>1.5$-fold reduction in side chain arginylation of Asp and Glu residues in COVID-19 patients (Figure 4A). Arginylation is an emerging poorly characterized modification, which is still non-routine during proteomics analysis and normally requires manual data validation. While it was not feasible to manually validate all identified peptides, we validated a number of representative MS/MS spectra (Dataset 1) to confirm that arginylation on the identified sites is likely.

Both arginylation and $\mathrm{Thr}$ phosphorylation have been previously proposed to play a global regulatory role. Thus, we performed further analysis of these two modifications to dissect the likely functional consequences of their change in COVID-19. Mapping the identified sites for these two modifications on the target proteins identified in our sets (see Supplementary Tables 

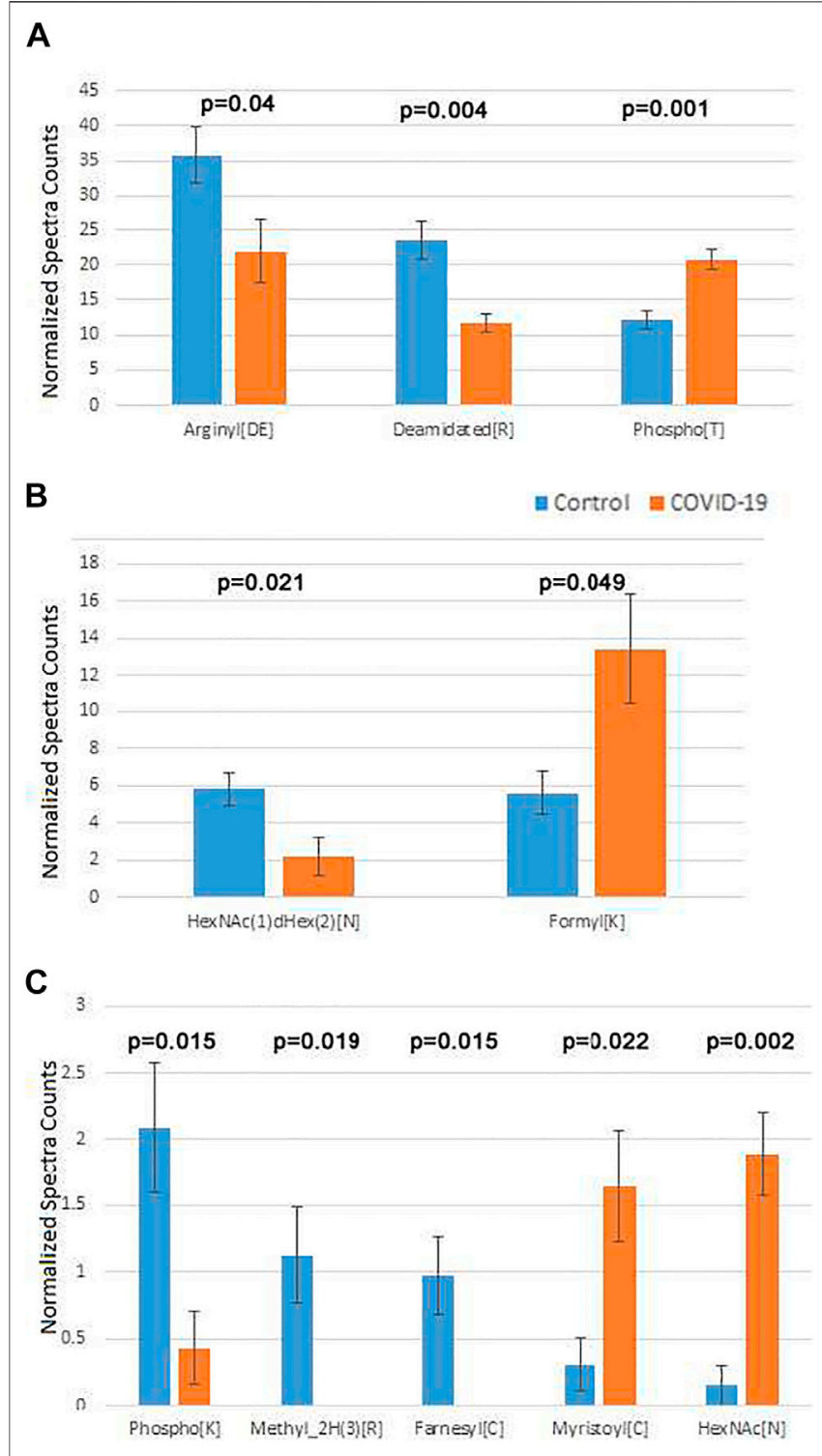

FIGURE 4 | Plasma proteins from COVID-19 patients exhibits changes in the overall levels of several physiological posttranslational modifications. Normalized spectra counts for high (A), intermediate (B), and low abundance (C) hits are plotted on different scales. Error bars represent SEM ( $n=7$ for control, 6 for COVID-19). $p$ values calculated by 2 -tailed Student's T-test are listed on top of each set of bars.

S8, S9 for arginylation and phosphorylation, respectively) revealed that in addition of the total level change for each of the PTMs, COVID-19 patients also exhibit a different repertoire of arginylation and phosphorylation sites compared to control. This points to a likely possibility that the target proteins affected by these altered regulatory PTMs, including components of the blood coagulation cascade and immune response, along with several other functions implicated in COVID-19 infection, may be differentially regulated by these two PTMs in healthy individuals versus those infected by SARS-CoV-2. While the effect of these PTMs is not yet understood, it is attractive to suggest that arginylation/phosphorylation on these sites may underlie COVID-19 dependent protein regulation and disease progression.

The two less abundant lists included a number of additional PTMs that play important physiological roles. Among these were two types of N-linked glycosylation: $\operatorname{HexNAc}(1) \mathrm{dHex}(2)[\mathrm{N}]$ was up in control (Figure 4, middle), and HexNAc [N], was up in COVID-19 (Figure 4, bottom). Notably, changes in glycosylation were previously observed on the viral SARS-CoV-2 proteins during COVID-19 (Praissman and Wells, 2021; Reis et al., 2021; Shajahan et al., 2021; Zhong et al., 2021), even though such changes in the host organism, to the best of our knowledge, were not previously reported. Additional modifications on this list were detected at very low levels. Even though physiologically important, it is difficult to assess the biological role of their changes in the plasma of COVID-19 patients.

In addition to these PTMs, a substantial number of amino acid substitutions were significantly different between COVID-19 and control plasma (Supplementary Figure S5). Of those, Glu to Asp substitution was by far the most abundant (Supplementary Figure S5, bottom). These substitutions normally reflect single nucleotide polymorphisms (SNPs), and thus are not a result of SARS-CoV-2 infection. However, the presence of these SNPs potentially reflect genetic changes that might make the patients more vulnerable to COVID-19.

\section{CONCLUSION}

To our knowledge, the data presented in this manuscript represents the first global proteome and peptidome analysis of plasma from COVID-19 patients. Other studies have extensively analyzed the proteomics and posttranslational state of SARSCoV-2 proteins during COVID-19 infection, but no one has as yet focused on the changes in the patient plasma proteome.

Our data suggests the existence of global protein-level trends in the patients' plasma that may inform our understanding of biology, prevention, diagnostics, and treatment of COVID-19. We find that plasma proteins in COVID-19 patients contain elevated levels of a limited number of proteins associated with inflammation and immune response, and some of these proteins, including fibrinogen, apparently undergo different proteolytic events compared to normal controls. Some of these changes may underlie the less understood clinical symptoms of COVID-19.

Our study shows that COVID-19 infection is accompanied by a prominent posttranslational signature, with differential increase and reduction in several key regulatory modifications, including glycosylation, citrullination, Thr phosphorylation, and arginylation. Decoding this signature may potentially inform our understanding of the biology, diagnostics, and treatment of COVID-19.

\section{MATERIALS AND METHODS}

\subsection{Biosafety}

All human patient samples were handled under BSL-2 containment. 


\subsection{Human Patient Samples}

Blood from 7 healthy controls and 6 COVID-19 patients was collected in lavender-top EDTA tubes from BD (cat no. 368661). These tubes come with $10.8 \mathrm{mg}$ K2 EDTA spray dried inside the tube. Blood was drawn into the tube by clinical staff and handed off to the processing unit within $8 \mathrm{~h}$, usually much less. Tubes were then spun down at $1,000 \mathrm{x} \mathrm{g}$ at room temperature for $15 \mathrm{~min}$. Plasma supernatant was removed, aliquoted, snap frozen and stored on dry ice for $\sim 1 \mathrm{~h}$, then transferred to $-80^{\circ} \mathrm{C}$ for storage.

\subsection{Plasma Fractionation for Protein and Peptide Fractions}

\subsubsection{Plasma Clarification}

Frozen plasma aliquots were thawed on ice and centrifuged at $13,000 \mathrm{~g}$ at $4^{\circ} \mathrm{C}$ for $30 \mathrm{~min}$. The supernatant was used for peptide isolation or further depleted of IgG and albumin for analyzing the protein composition using mass spectrometry.

\subsubsection{Peptide Isolation}

$50 \mu \mathrm{l}$ of clarified plasma was mixed with $200 \mu \mathrm{l}$ of Phosphate Buffered Saline. $1 \mathrm{ml}$ of freshly prepared $100 \mathrm{mM}$ Tris, $8 \mathrm{M}$ urea, $\mathrm{pH} 7.5$ at room temperature was used to denature the proteins and dissociate bound peptides from abundant plasma proteins. Denatured samples were loaded onto a pre-rinsed Amicon Ultra $30 \mathrm{kDa}$ MWCO filter (Millipore) and centrifuged at 13,000 $\mathrm{g}$ at $4^{\circ} \mathrm{C}$ for $20 \mathrm{~min}$. Formic acid was added to the flow-through containing primarily peptides to a final concentration of $0.1 \%$ (v/v). MacroSpin Vydac Silica C18 columns (the Nest Group, Inc., Part \#SMM SS18V, Lot \#060310) were pre-washed first with $500 \mu \mathrm{l}$ of $100 \%$ acetonitrile and then with water followed by centrifugation at $100 \mathrm{~g}$ at $4^{\circ} \mathrm{C}$ for $1 \mathrm{~min}$ to remove the liquid after each wash. The same centrifugation condition was used for all subsequent steps. Briefly, columns were equilibrated with $300 \mu \mathrm{l}$ $0.2 \%$ acetonitrile; the $30 \mathrm{kDA}$ MWCO flow-through was applied; columns were washed three times with $400 \mu \mathrm{l}$ of $0.1 \%$ formic acid, and peptides were then eluted using $300 \mu \mathrm{l}$ of $0.1 \%$ formic acid, $50 \%$ acetonitrile, snap frozen in liquid nitrogen, and stored at $-80^{\circ} \mathrm{C}$ until the analysis.

\subsubsection{Albumin \& IgG Depletion for Proteomics}

The plasma samples were depleted of IgG and albumin using Albumin \& IgG depletion SpinTrap columns (GE healthcare) according to manufacturer's instructions. Briefly, the column was resuspended, and the storage buffer was discarded by centrifugation at $100 \mathrm{~g}$ for $30 \mathrm{~s}$. The column was then equilibrated with $400 \mu \mathrm{l}$ of binding buffer ( $20 \mathrm{mM}$ sodium phosphate, $150 \mathrm{mM}$ sodium chloride, $\mathrm{pH}$ 7.4), which was discarded by centrifugation at $800 \mathrm{~g}$ for $30 \mathrm{~s}$. Immediately after thawing an aliquot, the plasma sample was diluted 1:1 with binding buffer, applied to the column, mixed with the resin and incubated for $5 \mathrm{~min}$ at room temperature. The albumin and IgG depleted protein fraction was collected by centrifugation at $800 \mathrm{~g}$ for $30 \mathrm{~s}$, the column was washed twice with $100 \mu \mathrm{l}$ binding buffer flowed by centrifugation at $800 \mathrm{~g}$ for $30 \mathrm{~s}$, and the flowthrough and washes were combined. The eluted proteins were precipitated by adding 9 volumes of $-20^{\circ} \mathrm{C}$ ethanol and stored overnight at $4^{\circ} \mathrm{C}$. Precipitated protein was collected by centrifugation at $13000 \mathrm{~g}$ at $4^{\circ} \mathrm{C}$ for $30 \mathrm{~min}$. The pellet was heat inactivated at $60^{\circ} \mathrm{C}$ for $20 \mathrm{~min}$ to denature any viral load that might have been present.

\subsection{Peptidomics}

\subsubsection{Sample Preparation and LC-MS/MS Analysis}

Total peptide fraction purified by $\mathrm{C} 18$ column from 13 COVID19 patient and control plasma (7 controls and 6 diseased) were lyophilized and resuspended in $30 \mathrm{ul}$ of $3 \%$ acetonitrile, $0.1 \%$ formic acid. The volume of plasma was kept constant between samples at each step to enable non-normalized comparison of abundances for each identified peptide. $5 \mu \mathrm{l}$ of each sample was analyzed by LC-MS/MS on the Thermo Q Exactive HF mass spectrometer using a 2-h LC gradient (Su et al., 2021).

\subsubsection{Data Analysis}

MS/MS data were analyzed using Thermo Proteome Discoverer v2.4. Spectra were searched using no-enzyme specificity against the UniProt human proteome database (10/02/2020) and a common contaminant database using Sequest HT. Percolator target False Discovery Rate was set at 0.01 (Strict) and 0.05 (Relaxed). Only peptides identified with high confidence were retained. Common contaminants (primarily keratins) were removed. Peptide abundance values were determined from the peptide chromatographic peak areas. Ratio, $p$-value (t-test) and adjusted q-value ( $p$-value adjusted to account for multiple testing using Benjamini-Hochberg FDR) were calculated using the non-normalized abundance values. Significant changing peptides are defined as peptides with a minimum absolute fold change of 2 , adjusted $p$-value of 0.05 , and identified in a minimum of 3 replicates in either group.

To produce the charts shown in the main and supplemental figures, we summed up total abundances of all peptides belonging to each protein that exhibited significant differences between groups and calculated the average of these sums between all control and all COVID-19 samples. For the chart showing differential proteolytic patterns of fibrinogen and serglycin (Figure 2), abundances of individual peptides were averaged across samples and plotted against each peptide sequence.

\subsection{Proteomics}

\subsubsection{Sample Preparation and LC-MS/MS Analysis}

The ethanol precipitated albumin and IgG depleted COVID-19 patient plasma ( 7 controls, denoted by 3 -digit numbers starting with 0 in the supplemental tables, and 6 diseased, denoted by 3 digit numbers starting with 5 in the supplemental tables) fractions were dissolved in $50 \mu \mathrm{l}$ of $1 \%$ SDS, $50 \mathrm{mM}$ Tris-Cl pH 7.5.10 $\mu \mathrm{l}$ of each was run into a NuPAGE $10 \%$ Bis-Tris gel (Thermo Scientific) for a short distance. The entire stained gel regions were excised, reduced with tris(2-carboxyethyl)phosphine (TCEP), alkylated with iodoacetamide, and digested with trypsin. Tryptic digests were analyzed using a single-shot extended $4 \mathrm{~h}$ LC gradient on the Thermo Q Exactive Plus mass spectrometer.

\subsubsection{Data Analysis}

Peptide sequences were identified using MaxQuant 1.6.17.0 (Cox and Mann, 2008). MS/MS spectra were searched against a UniProt human proteome database $(10 / 02 / 2020)$ and a common 
contaminants database using full tryptic specificity with up to two missed cleavages, static carboxamidomethylation of Cys, and variable Met oxidation, protein N-terminal acetylation and Asn deamidation. "Match between runs" feature was used to help transfer identifications across experiments to minimize missing values. Consensus identification lists were generated with false discovery rates set at $1 \%$ for protein and peptide identifications. Statistical analyses were performed using Perseus 1.6.15.0 (Tyanova et al., 2016). Protein fold changes were determined from the Intensity values. Missing values were imputed with a minimum Intensity value, and t-test $p$-values were adjusted to account for multiple testing using the permutation-based FDR function in Perseus. High confidence identification of proteins with significant change was determined based on the following criteria: minimum absolute fold change of 2 , q-value $<0.05$, identified by a minimum of 2 razor + unique peptides, and detected in at least 3 of the replicates in one of the groups compared.

\subsection{Posttranslational Modifications Analysis}

The "Proteomics" MS/MS data described above were analyzed using pFind 3.1.5 (Chi et al., 2018). Two separate pFind searches were performed: Control (containing all 7 control samples) and Disease (containing all 6 samples). Spectra were searched using partial tryptic specificity against the UniProt human proteome database (10/02/2020). The "Open Search" option was used to identify PTMs. Data were filtered using a precursor mass tolerance of $10 \mathrm{ppm}$, fragment ion tolerance of $20 \mathrm{ppm}$, and FDR $<1 \%$ at peptide and protein level.

\section{DATA AVAILABILITY STATEMENT}

The data presented here has been deposited into the MassIVE public repository (https://massive.ucsd.edu/ProteoSAFe/static/ massive.jsp) with the accession MSV000088382, and the ProteomeXchange repository (http://www.proteomecentral. proteomexchange.org/cgi/) with the accession PXD029756.

\section{ETHICS STATEMENT}

The studies involving human participants were reviewed and approved by the University of Pennsylvania Institutional Review Board. Written informed consent for participation was not

\section{REFERENCES}

Ahmed, S., Zimba, O., and Gasparyan, A. Y. (2020). Thrombosis in Coronavirus Disease 2019 (COVID-19) through the Prism of Virchow's Triad. Clin. Rheumatol. 39 (9), 2529-2543. doi:10.1007/s10067-020-05275-1

Anand, P., Puranik, A., Aravamudan, M., Venkatakrishnan, A., and Soundararajan, V. (2020). SARS-CoV-2 Strategically Mimics Proteolytic Activation of Human ENaC. Elife 9. doi:10.7554/eLife.58603

Arapidi, G., Osetrova, M., Ivanova, O., Butenko, I., Saveleva, T., Pavlovich, P., et al. (2018). Peptidomics Dataset: Blood Plasma and Serum Samples of Healthy Donors Fractionated on a Set of Chromatography Sorbents. Data in Brief 18, 1204-1211. doi:10.1016/j.dib.2018.04.018 required for this study in accordance with the national legislation and the institutional requirements.

\section{AUTHOR CONTRIBUTIONS}

PV, AK, H-YT, and DS designed the research. PV and H-YT performed the experiments and analyzed data. AK and DS analyzed data and wrote the paper.

\section{FUNDING}

The Human Immunology Core is funded in part by NIH P30AI0450080 and P30-CA016520. This work was supported by the NIH Grant R35GM122505 to AK and R50 CA221838 to $\mathrm{H}-\mathrm{YT}$.

\section{ACKNOWLEDGMENTS}

We are grateful to Dr. John Wherry and Penn Immune Health Initiative for support of this work and providing the plasma samples for this analysis. We thank the Human Immunology Core (HIC) facility for assistance with sample processing and provision of control samples for this study. The UPenn COVID Processing Unit is a unit of individuals from diverse laboratories at the University of Pennsylvania who volunteered time and effort to enable study of COVID-19 patients during the pandemic: Zahidul Alam, Mary M. Addison, Amy E. Baxter, Katelyn T. Byrne, Aditi Chandra, Kurt D’Andrea, Hélène C. Descamps, Jeanette Dougherty, Allison R. Greenplate, Nicholas Han, Yaroslav Kaminskiy, Justin Kim, Oliva Kuthuru, Jacob T. Hamilton, Julia Han Noll, Dalia K. Omran, Ajinkya Pattekar, Eric Perkey, Nils Wellhausen, Elizabeth M. Prager, Dana Pueschl, Jennifer B. Shah, Jake S. Shilan, and Ashley N. Vanderbeck.

\section{SUPPLEMENTARY MATERIAL}

The Supplementary Material for this article can be found online at: https://www.frontiersin.org/articles/10.3389/fcell.2022.807149/ full\#supplementary-material

Chi, H., Liu, C., Yang, H., Zeng, W.-F., Wu, L., Zhou, W.-J., et al. (2018). Comprehensive Identification of Peptides in Tandem Mass Spectra Using an Efficient Open Search Engine. Nat. Biotechnol. 36, 1059-1061. doi:10.1038/nbt. 4236

Cox, J., and Mann, M. (2008). MaxQuant Enables High Peptide Identification Rates, Individualized p.p.b.-Range Mass Accuracies and Proteome-Wide Protein Quantification. Nat. Biotechnol. 26 (12), 1367-72. doi:10.1038/nbt.1511

Gómez-Mesa, J. E., Galindo-Coral, S., Montes, M. C., and Muñoz Martin, A. J. (2021). Thrombosis and Coagulopathy in COVID-19. Curr. Probl. Cardiol. 46 (3), 100742. doi:10.1016/j.cpcardiol.2020.100742

Gorski, J. P., Hugli, T. E., and Muller-Eberhard, H. J. (1979). C4a: The Third Anaphylatoxin of the Human Complement System. Proc. Natl. Acad. Sci. 76 (10), 5299-5302. doi:10.1073/pnas.76.10.5299 
Hanff, T. C., Mohareb, A. M., Giri, J., Cohen, J. B., and Chirinos, J. A. (2020). Thrombosis in COVID -19. Am. J. Hematol. 95 (12), 1578-1589. doi:10.1002/ ajh. 25982

Harapan, B. N., and Yoo, H. J. (2021). Neurological Symptoms, Manifestations, and Complications Associated with Severe Acute Respiratory Syndrome Coronavirus 2 (SARS-CoV-2) and Coronavirus Disease 19 (COVID-19). J. Neurol. 268 (9), 3059-3071. doi:10.1007/s00415-021-10406-y

Katneni, U. K., Alexaki, A., Hunt, R. C., Schiller, T., DiCuccio, M., Buehler, P. W., et al. (2020). Coagulopathy and Thrombosis as a Result of Severe COVID-19 Infection: A Microvascular Focus. Thromb. Haemost. 120 (12), 1668-1679. doi:10.1055/s-0040-1715841

Li, D., Fu, Y., Sun, R., Ling, C. X., Wei, Y., Zhou, H., et al. (2005). pFind: A Novel Database-Searching Software System for Automated Peptide and Protein Identification via Tandem Mass Spectrometry. Bioinformatics 21 (13), 3049-3050. doi:10.1093/bioinformatics/bti439

May, J. E., Wolberg, A. S., and Lim, M. Y. (2021). Disorders of Fibrinogen and Fibrinolysis. Hematology/Oncology Clin. North America 35, 1197-1217. doi:10.1016/ j.hoc.2021.07.011

Meyer, B., Chiaravalli, J., Gellenoncourt, S., Brownridge, P., Bryne, D. P., Daly, L. A., et al. (2021). Characterising Proteolysis during SARS-CoV-2 Infection Identifies Viral Cleavage Sites and Cellular Targets with Therapeutic Potential. Nat. Commun. 12 (1), 5553. doi:10.1038/s41467-021-25796-w

Montenegro, F., Unigarro, L., Paredes, G., Moya, T., Romero, A., Torres, L., et al. (2021). Acute Respiratory Distress Syndrome (ARDS) Caused by the Novel Coronavirus Disease (COVID-19): A Practical Comprehensive Literature Review. Expert Rev. Respir. Med. 15 (2), 183-195. doi:10.1080/17476348. 2020.1820329

Novelli, G., Biancolella, M., Mehrian-Shai, R., Colona, V. L., Brito, A. F., Grubaugh, N. D., et al. (2021). COVID-19 One Year into the Pandemic: From Genetics and Genomics to Therapy, Vaccination, and Policy. Hum. Genomics 15 (1), 27. doi:10. 1186/s40246-021-00326-3

Østergaard, L. (2021). SARS CoV-2 Related Microvascular Damage and Symptoms during and after COVID-19: Consequences of Capillary Transit-time Changes, Tissue Hypoxia and Inflammation. Physiol. Rep. 9 (3), e14726. doi:10.14814/phy2. 14726

Praissman, J. L., and Wells, L. (2021). Proteomics-Based Insights into the SARS-CoV-2Mediated COVID-19 Pandemic: A Review of the First Year of Research. Mol. Cell Proteomics 20, 100103. doi:10.1016/j.mcpro.2021.100103

Ramos-Guzmán, C. A., Ruiz-Pernía, J. J., and Tuñón, I. (2020). Unraveling the SARSCoV-2 Main Protease Mechanism Using Multiscale Methods. ACS Catal. 10, 12544-12554. doi:10.1021/acscatal.0c03420

Reis, C. A., Tauber, R., and Blanchard, V. (2021). Glycosylation Is a Key in SARS-CoV-2 Infection. J. Mol. Med. 99 (8), 1023-1031. doi:10.1007/s00109-021-02092-0

Saha, S., Wong, C. C. L., Xu, T., Namgoong, S., Zebroski, H., Yates, J. R., et al. (2011). Arginylation and Methylation Double up to Regulate Nuclear Proteins and Nuclear Architecture In Vivo. Chem. Biol. 18 (11), 1369-1378. doi:10.1016/j.chembiol.2011. 08.019

Schmulson, M., Dávalos, M. F., and Berumen, J. (2020). Beware: Gastrointestinal Symptoms Can Be a Manifestation of COVID-19. Revista de Gastroenterología de México (English Edition) 85 (3), 282-287. doi:10.1016/j.rgmxen.2020.04.001

Scully, O. J., Chua, P.-J., Harve, K. S., Bay, B.-H., and Yip, G. W. (2012). Serglycin in Health and Diseases. Anat. Rec. 295 (9), 1415-1420. doi:10.1002/ar.22536

Shajahan, A., Pepi, L. E., Rouhani, D. S., Heiss, C., and Azadi, P. (2021). Glycosylation of SARS-CoV-2: Structural and Functional Insights. Anal. Bioanal. Chem. 413, 7179-7193. doi:10.1007/s00216-021-03499-x

Stockley, R. A. (2014). Alpha1-antitrypsin Review. Clin. Chest Med. 35 (1), 39-50. doi:10.1016/j.ccm.2013.10.001

Su, C., Lu, F., Soldan, S. S., Lamontagne, R. J., Tang, H.-Y., Napoletani, G., et al. (2021). EBNA2 Driven Enhancer Switching at the CIITA-DEXI Locus Suppresses HLA Class II Gene Expression during EBV Infection of
B-Lymphocytes. Plos Pathog. 17 (8), e1009834. doi:10.1371/journal.ppat. 1009834

Swelum, A. A., Shafi, M. E., Albaqami, N. M., El-Saadony, M. T., Elsify, A., Abdo, M., et al. (2020). COVID-19 in Human, Animal, and Environment: A Review. Front. Vet. Sci. 7, 578. doi:10.3389/fvets.2020.00578

Świderek, K., and Moliner, V. (2020). Revealing the Molecular Mechanisms of Proteolysis of SARS-CoV-2 Mproby QM/MM Computational Methods. Chem. Sci. 11 (39), 10626-10630. doi:10.1039/d0sc02823a

Tang, T., Jaimes, J. A., Bidon, M. K., Straus, M. R., Daniel, S., and Whittaker, G. R. (2021). Proteolytic Activation of SARS-CoV-2 Spike at the S1/S2 Boundary: Potential Role of Proteases beyond Furin. ACS Infect. Dis. 7 (2), 264-272. doi:10.1021/acsinfecdis.0c00701

Troyer, E. A., Kohn, J. N., and Hong, S. (2020). Are We Facing a Crashing Wave of Neuropsychiatric Sequelae of COVID-19? Neuropsychiatric Symptoms and Potential Immunologic Mechanisms. Brain Behav. Immun. 87, 34-39. doi:10. 1016/j.bbi.2020.04.027

Tyanova, S., Temu, T, Sinitcyn, P., Carlson, A., Heinn, M. Y., Geiger, T., et al. (2016). The Perseus Computational Platform for Comprehensive Analysis of (Prote)omics Data. Nat Methods. 13 (9), 731-40. doi:10.1038/nmeth.3901

Vakil-Gilani, K., and O'Rourke, K. (2020). Are Patients with Rheumatologic Diseases on Chronic Immunosuppressive Therapy at Lower Risk of Developing Severe Symptoms when Infected with COVID-19. Clin. Rheumatol. 39 (7), 2067-2068. doi:10.1007/s10067-020-05184-3

Wang, J., Han, X., Wong, C. C. L., Cheng, H., Aslanian, A., Xu, T., et al. (2014). Arginyltransferase ATE1 Catalyzes Midchain Arginylation of Proteins at Side Chain Carboxylates In Vivo. Chem. Biol. 21 (3), 331-337. doi:10.1016/j. chembiol.2013.12.017

Wang, J., Yates, J. R., 3rd, and Kashina, A. (2019). Biochemical Analysis of Protein Arginylation. Methods Enzymol. 626, 89-113. doi:10.1016/bs.mie. 2019.07.028

Wang, L.-h., Li, D.-Q., Fu, Y., Wang, H.-P., Zhang, J.-F., Yuan, Z.-F., et al. (2007). pFind 2.0: A Software Package for Peptide and Protein Identification via Tandem Mass Spectrometry. Rapid Commun. Mass. Spectrom. 21 (18), 2985-2991. doi:10.1002/rcm.3173

Zheng, H., Li, H., Zhang, J., Fan, H., Jia, L., Ma, W., et al. (2020). Serum Amyloid A Exhibits pH Dependent Antibacterial Action and Contributes to Host Defense against Staphylococcus aureus Cutaneous Infection. J. Biol. Chem. 295 (9), 2570-2581. doi:10.1074/jbc.RA119.010626

Zhong, L., Zhu, L., and Cai, Z.-W. (2021). Mass Spectrometry-Based Proteomics and Glycoproteomics in COVID-19 Biomarkers Identification: A Mini-Review. J. Anal. Test. 5, 298-313. doi:10.1007/ s41664-021-00197-6

Conflict of Interest: The authors declare that the research was conducted in the absence of any commercial or financial relationships that could be construed as a potential conflict of interest.

Publisher's Note: All claims expressed in this article are solely those of the authors and do not necessarily represent those of their affiliated organizations, or those of the publisher, the editors and the reviewers. Any product that may be evaluated in this article, or claim that may be made by its manufacturer, is not guaranteed or endorsed by the publisher.

Copyright (c) 2022 Vedula, Tang, Speicher and Kashina. This is an open-access article distributed under the terms of the Creative Commons Attribution License (CC BY). The use, distribution or reproduction in other forums is permitted, provided the original author(s) and the copyright owner(s) are credited and that the original publication in this journal is cited, in accordance with accepted academic practice. No use, distribution or reproduction is permitted which does not comply with these terms. 